\title{
MICROMIRROR ARRAY PHASE MODULATOR FOR ULTRASHORT OPTICAL PULSE SHAPING
}

\author{
Kebin Li, Uma Krishnamoorthy, Jonathan P. Heritage \\ Department of Electrical Engineering, University of California Davis, Davis, CA 95616 \\ Olav Solgaard \\ Department of Electrical Engineering, Stanford University, Stanford, CA 94035
}

\begin{abstract}
A linear phased array of programmable micromirrors provides dual mode motion. The micromirrors can be individually rotated about an axis through a mirror, and the whole mirror can be translated perpendicular to the mirror surface. Rotational motion adds the flexibility of constructing piecewise linear approximations to a desired phase modulation profile, enabling spatial phase modulation with higher diffraction efficiency than achievable with only translation. A new interferometer using onchip stationary reference micromirror to calibrate the motion of the movable micromirror is presented. The coherent behavior of the micromirror array is also demonstrated. Applications include spatial scanning, and ultrashort-pulse shaping.
\end{abstract}

\section{INTRODUCTION}

Over the past decade powerful optical waveform synthesis (or pulse shaping) methods have been developed which allow generation of complicated ultrafast optical waveforms according to user specification. Coupled with the recent advances and resulting widespread availability of femtosecond lasers, as well as improvements in femtosecond pulse characterization techniques, femtosecond pulse shaping is poised to impact many diverse applications. Those areas include quantum control with light, laser selective chemistry, particle acceleration, coherent control over ultrafast physical processes, high field physics and optical communication.

Pulse shaping using reflective spatial light phase modulator (SLPM) is shown in Fig. 1. An ultrashort laser pulse is incident on a simple grating and lens apparatus, separated by the focal length of the lens, which spatially decomposes the pulse into individual optical frequency components at the focal plane of lens. One can insert spatially patterned masks or a programmable SLPM at the focal plane in order to manipulate the amplitude and the phase of the spatially dispersed optical frequency components. After the various frequencies are reflected and reassembled into a single

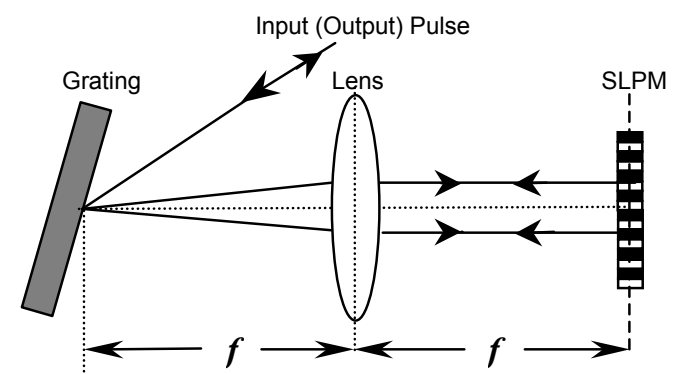

Figure 1. Reflective ultrashort pulse-shaping apparatus.

collimated beam by the lens and grating, one obtains a shaped pulse. In the high resolution limit, the pulse shape is determined by the Fourier transform of the amplitude and phase pattern imposed on the spectrum by the masks. The pulse shaper is dispersion free, so that in the presence of a flat mirror, the output and input pulses are identical.

Programmable SLPM are a key component in a femtosecond pulse shaper [1] and are thus under intense investigation recently because of its potential applications. Existing linear SLPMs, fall into two categories; deformable continuous thin membrane mirrors [2], and pixilated arrays of liquid crystal phase shifters [3].

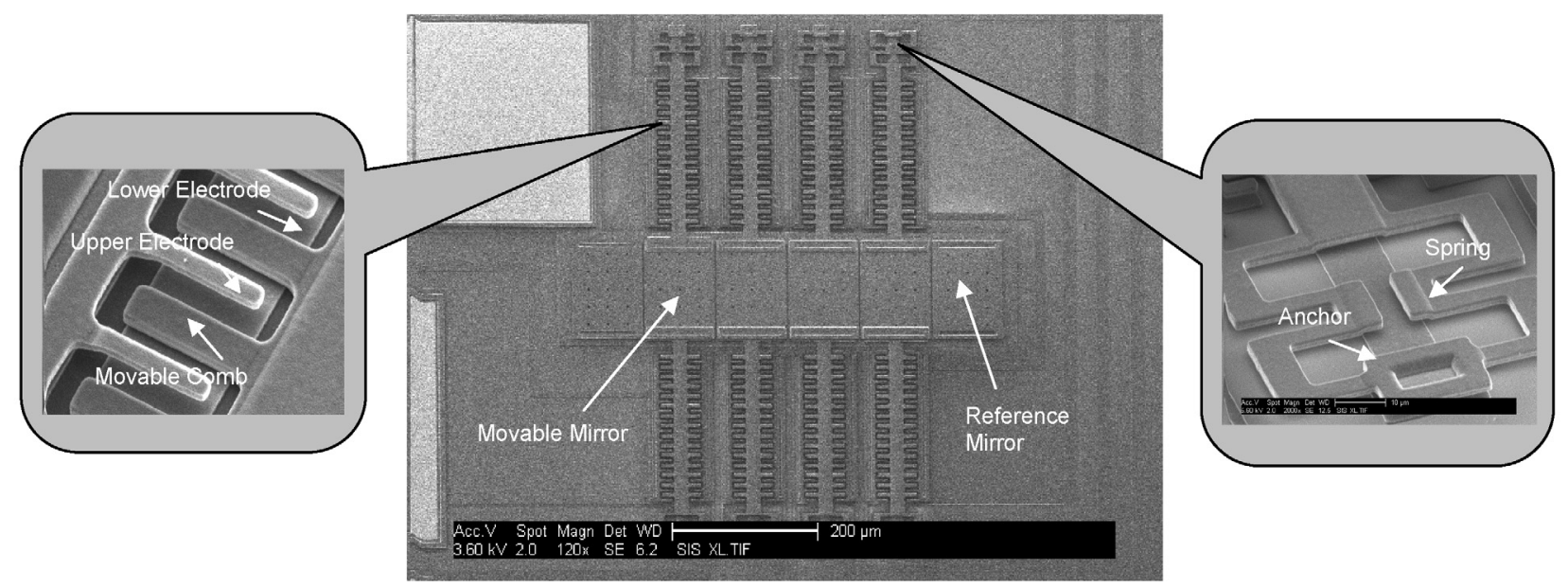

Figure 2. SEM photographs of the micromirror array showing four movable mirrors and two stationary reference mirrors. The left blowup shows the details of the upper comb electrode and the lower planer capacitive electrode. The right blowup shows the tether and spring that support the mirror.

Travel support has been generously provided by the Transducers Research

Foundation and by the DARPA MEMS and DARPA BioFlips Programs 
Deformation of a continuous membrane produces smooth spectral phase variations and a corresponding, high quality optical pulse but membranes are not capable of producing localized rapid spectral phase changes. Pixilated arrays of phase modulators can produce rapid phase changes between pixels. However, the phase shift for each pixel is constant and the resulting discrete approximation to smooth phase variations results in undesired energy in the pulse wings.

Here we introduce a new coherent micromirror-array SLPM (Fig.2), which is fabricated by the MUMPS process. The mirrors of the array have both rotational motion and elevation control [4] enabling high fidelity phase modulation. Vertical elevation (modulo $\pi$ ) of the mirror introduces the desired phase shift for each pixel, while mirror tilt allows a much-improved piecewise linear approximation to the local phase variation compared to commercially-available SLPMs, such as liquid crystal and thin membrane mirror phase modulators.

\section{MICROMIRROR DESIGN AND FABRICATION}

In order to achieve both rotation and linear motion of the micromirror, we designed an actuator with two upper vertical comb drives and two lower planar capacitive drives. Figure 2 shows an SEM micrograph of an array fabricated in the MUMPS surface micromachining process. In our SLPM design, each mirror is $100 \times 120$ microns and has four stationary electrodes on each side. A pair of vertical comb drives emanating from each mirror, are tethered their far ends. They act as springs to support a freely floating mirror. Applying the same potential to each of the fixed upper vertical combs raises a mirror with out applying a torque. A mirror may be similarly depressed by applying the same potential on each of four lower planar conductors. A mirror is rotated by applying a potential to one comb (on both sides of the mirror) with another equal potential is on the diagonally opposing planar electrode. Ideally, the applied potentials are arranged such that the forces on the torsion bar are equal and opposite and then no vertical motion accompanies the rotation. Unbalanced forces result in both vertical motion and rotation.

Surface flatness of the micromirror is very important for the optical applications. We use a Zygo system to measure the surface planarity of our micromirror array. Figure 3 shows surface plots of two micromirrors in the array. The phase change across each mirror is less than one hundredth of $2 \pi$ for 1.6 micron input pulse
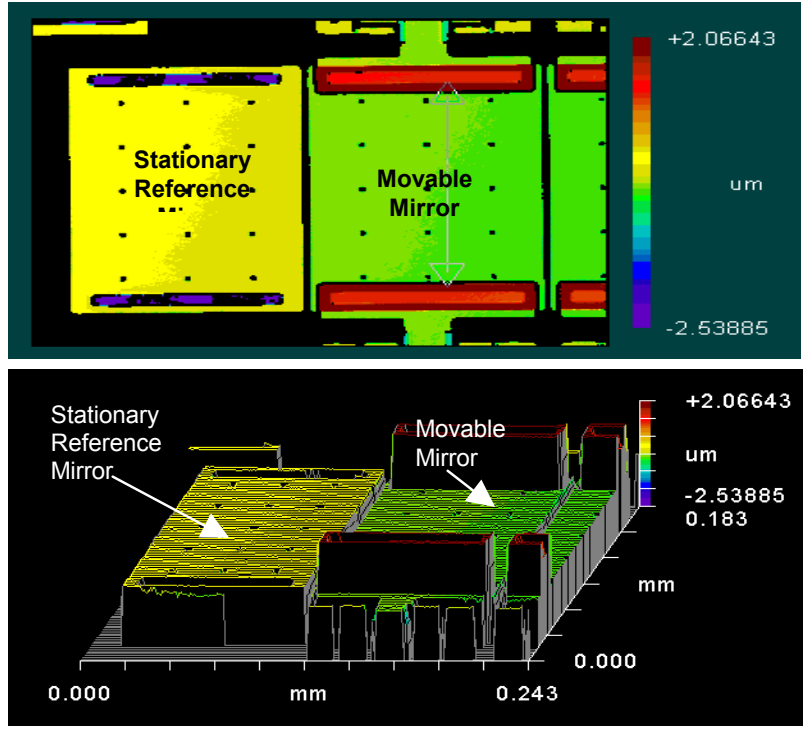

Figure 3. Micromirror surface flatness measurement using Zygo system, radius of curvature reaches as much as $4.8 \mathrm{~m}$ for $100 \mu m \times 120 \mu m$ mirrormirror

central wavelength. The radius of curvature perpendicular to the comb drive direction is 0.41 meter, and 4.8 meter in the direction of the drives. These polysilicon micromirrors, which are not coated, have 40 percent reflectivity. Although with gold coating, much higher reflectivity can be reached however, mirror flantens will be affected.

\section{ON-CHIP REFERENCE MIRROR INTERFEROMETER}

We designed a simple interferometer in order to calibrate the motions of the micromirrors. Reflections off the movable mirror under test and off an on-chip stationary reference mirror generate an interference pattern in the far field. Using a thin glass plate we split a laser beam into two coherent beams, which are focused on the two mirrors separately. The reflected far field interference pattern contains the information of movable micromirror position. This interferometer is less sensitive to vibrations than more

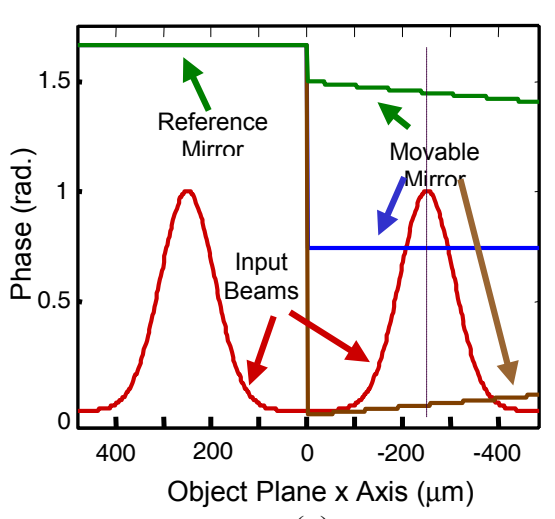

(a)

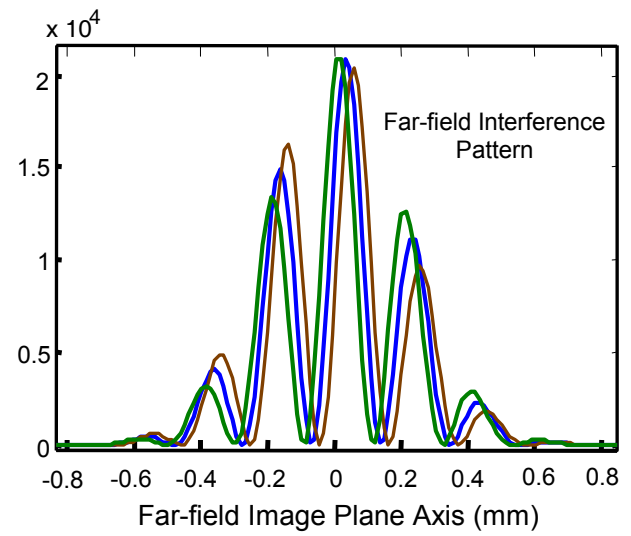

(b)

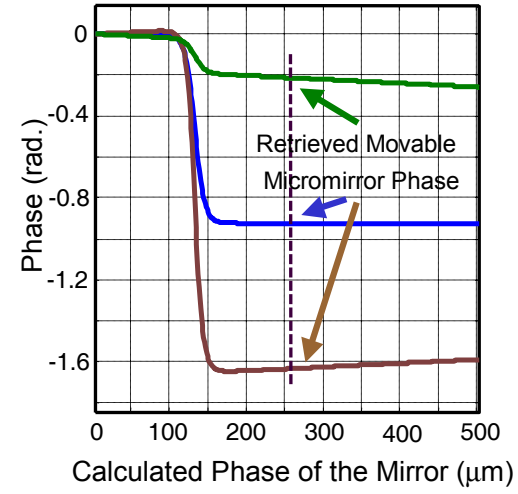

(c)

Figure 4. Simulation of the phase retrieval from the far field interference pattern created by the reflections from the reference and movable mirrors. (a) The three different states of the mirrors with the two coherent inputs overlaid. (b) Three interference patterns corresponding to the three positions of the movable mirror. (c) Movable-mirror phase calculated from the interference pattern. 
complicated interferometers with off-chip reference arms and is easy to operate.

This Fourier-transform method can be proved and simulated as shown in the following discussion. Assume two Gaussian coherent beams are focused, one on the reference mirror and the other on the movable micromirror. The spacing is $2 x_{0}$. The movable mirror tilts with a slope of $f_{0}$. The output field amplitude from the micromirrors is:

$$
g(x)=A e^{-a\left(x+x_{0}\right)^{2}}+A e^{-a\left(x-x_{0}\right)^{2}} e^{i 2 \pi f_{0}\left(x-x_{0}\right)} .
$$

The far field interference pattern field amplitude is the Fourier transform of the Eq.(1). The corresponding intensity is then found. Next, take the Fourier transform of the intensity profile of the interference pattern and make use of the positive component of the spectrum:

$$
C\left\{\cos \left[2 \pi f_{0}\left(x-2 x_{0}\right)\right]+i \sin \left[2 \pi f_{0}\left(x-2 x_{0}\right)\right]\right\},
$$

$C$ is a constant. It is easy to see now that if we divide the imaginary part by the real part and take the inverse tangent of that, one retrieves the slope, $f_{0}$, for the movable micromirror, when $x$ is near $2 x_{0}$.

We developed a simulation program to illustrate the process of phase retrieval. The simulation results in Fig.4 show how the spatial phase variation of the movable mirror (Fig. 4a) leads to a shift and intensity change of the far-field interference pattern (Fig. $4 b)$, from which the vertical displacement and tilt of the mirror under test can be retrieved (Fig. 4c).

\section{MICROMIRROR MOTION CALIBRATION}

The micromirror motion calibration can be put into three categories. There are, elevation or depression, tilt angle, and general motion with respect to applied voltage. An experimental measurement for the first two cases is shown in Fig. 5.

We previously reported [5] a special case of our interfrometer, which uses only one optical beam, and thus can only measure tilt and elevation change in separate measurements. Using a single spot, which covers two adjacent mirrors, an interference dip appears in the far field diffraction pattern. Changes its position are in proportion to the optical phase difference between the optical paths. When the mirrors are at the same height, constructive interference produces a single broad spot as is displayed in the farleft image in Fig. 5(a). When the mirror is elevated by $h=\lambda / 4$, the optical path length difference between the two mirrors results in destructive interference, as is displayed in the far right hand image in Fig. 5(a). The corresponding optical phase difference is then

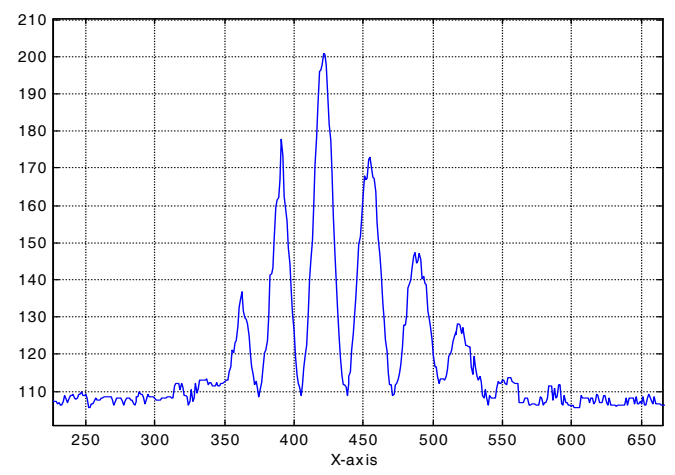

(a)

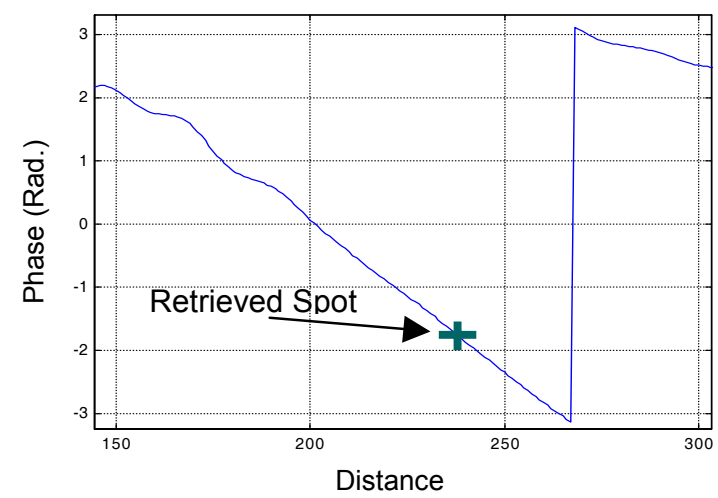

(b)

Figure 6. Experimentally measured far-field interference pattern and the retrieved phase of the movable mirror. (a) The far field interference fringe pattern generated by movable mirror and reference mirror. (b) Retrieved phase of the movable mirror. Both the vertical displacement and tilt angle can be calibrated. For example: The phase difference of the retrieved spot relative to the reference mirror is 4.75 radians, and the micromirror tilt angle is $0.33^{0}$

$4 \pi h / \lambda$. The phase shift for an arbitrary $h$ produces an off center interference dip as is displayed in the middle image of Fig. 5(a). Figure 5(b) shows the levitation displacement versus the applied potential to upper and lower electrodes. The lower electrodes, in
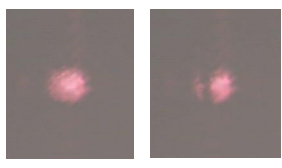

(a)

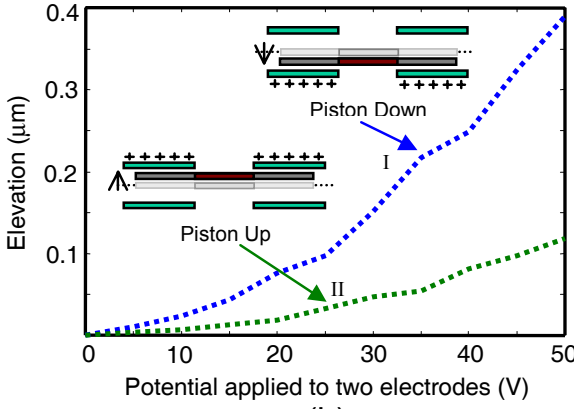

(b)

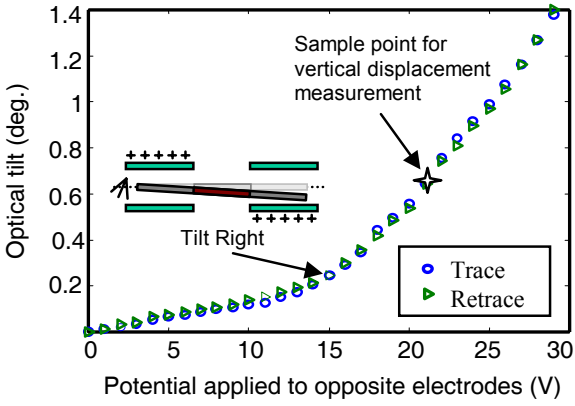

(c)

Figure 5. Measured micromirror motion. (a) CCD images of far field diffraction pattern obtained when both a movable mirror and a reference mirror are illuminated. Elevation increases from zero to $\lambda / 4$ from left to right. (b) Elevation vs. applied potentials for downward (curve I) and upward (curve II) displacements. (c) Tilt vs. applied potentials. The same potential is applied to the diagonally opposing electrodes. 
this design, provide greater force per unit applied potential than the comb structure. Reduction of electrode size allows design of symmetric, or alternatively, symmetric comb drives could be fabricated.

Figure 5(c) shows mirror tilt when the same potential is put on opposite electrodes. The measured useable static range of angular and vertical motions was 1.4 degrees (optical) and 1.2 microns, respectively. Because of the asymmetry of the electrodes, an elevation change occurs in this situation as well. We pick a sample point shown in Fig. 5(c) to measure the vertical shift caused by this asymmetry using our general Fourier-transform approach discussed before. Figure 6(a) shows the intensity profile of far field interference pattern measured by CCD camera when movable mirror is under this sample condition, 21.5 volts on opposite electrodes. The retrieved phase of the mirror is shown in Fig. 6(b). From that we get mirror tilt angle $0.33^{\circ}$ that matches well with the optical tilt $0.65^{\circ}$ in Fig. 5(c) using simple beam shift method.

We also retrieve vertical down shift 4.75 radians from the plot. It is shown in Fig. 3 that the stationary reference micromirror is 0.2 $\mu \mathrm{m}$ higher than the resting movable mirror due to the design. Since the coherent light source is $\lambda=0.632 \mu \mathrm{m}$, the phase delay in this case is 3.98 radians. So the actual mirror vertical down shift is 0.39 radians or $0.04 \mu \mathrm{m}$. Referring to the Fig. 5(b), we see that the actual elevation lies between curves II and I. This is the expected result which demonstrates the important capability to obtain both rotation and elevation in a single measurement.

\section{COHERENT PHASED ARRAY DEMONSTRATION}

To demonstrate the operation of the coherent micromirror array, we displayed the far field diffraction when illuminated with a monochromatic line source $(\lambda=632 \mathrm{~nm})$. This configuration is formally analogous to high-resolution femtosecond pulse shaping. We investigated three arrangements of the mirror array that illustrate coherent control of the diffracted wave front. Figure 7(a) shows three different mirror position patterns. Pattern 1 (light gray outline) is for free floating mirrors with no applied potential. This establishes the system diffraction limit as shown in Fig. 7(b), dotted peak. In pattern 2 (hashed diagonals) all the micromirrors tilt the same angle as a reference bulk mirror. This configuration produces a four-micromirror array, which is now a blazed diffraction grating. Figure 7(c) shows four diffraction orders plus the specular reflection. In sharp contrast, if we adjust each mirror height as shown in Fig. 7(a) the grating becomes the equivalent of a single, large tilted reference bulk mirror, except now, it is pixelated. Pattern 3, (gray fill) reflects only into a new specular direction, as shown in Fig. 7(b). Higher order diffraction is effectively suppressed. This operation can only be accomplished with a coherent array of mirrors that have simultaneous tilt and elevation.

\section{CONCLUSIONS}

In summary, we propose and demonstrate a new type of digitized programmable SLPM using MEMS technology. We have designed, fabricated, and calibrated array of micromirrors with independent tilt and elevation control, and demonstrated that our arrays can perform coherent phase modulation. These devices, when expanded to larger array sizes, will find applications in femtosecond pulse shaping.

\section{REFERENCES}

1. A.M. Weiner, J.P. Heritage and E. M.Kirschner, "Highresolution femtosecond pulse shaping," J. Opt. Soc. Amer., vol. B5, p.1563, 1988.

2. C. Dorrer, F. Salin, F. Verluise and J.P. Huignard, "Programmable phase control of femtosecond pulses by use of a nonpixelated spatial light modulator," Opt.Lett., vol. 23, (no.9), p709. May 1, 1998.

3. A.M. Weiner, D.E. Leaird, J.S. Patel and J.R. Wullert, "Programmable femtosecond pulse shaping by use of a multielement liquid-crystal phase modulator," Opt. Lett., vol. 15, p.326, 1990.

4. U. Krishnamoorthy, K.Li, K. Yu, D. Lee, J.P. Heritage and O. Solgaard, $11^{\text {th }}$ International Conference on Solid-State Sensors and Actuators (Transducer '01), Munich, Germany, June 10-14, 2001.

5. K. Li, U. Krishnamoorthy, J. P. Heritage, and O. Solgaard, "Coherent Micromirror arrays," Opt. Lett., vol 27, p.366, 2002.

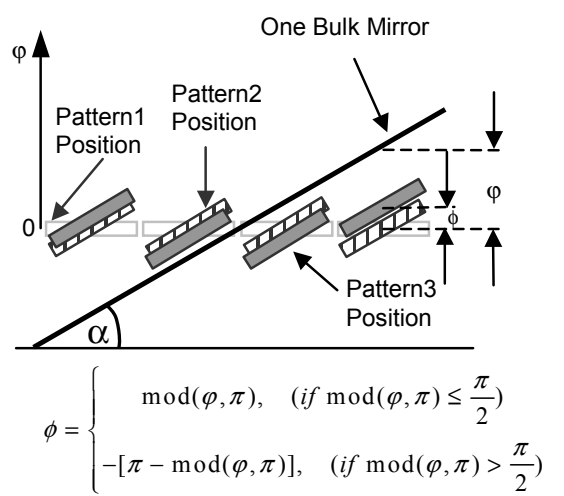

Positive $\phi$ for piston up, negative $\phi$ for piston down Maximum vertical displacement less then $\pi / 2$ or $\lambda / 4$

(a)

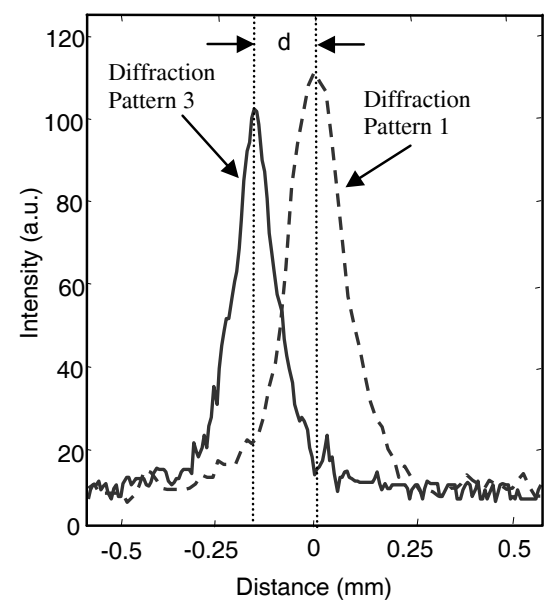

(b)

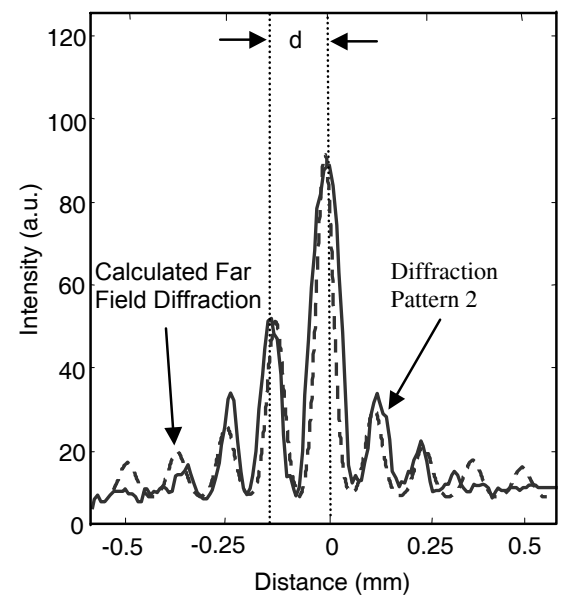

(c)

Figure 7. Coherent phased array far field diffraction patterns. (a) Three different mirror array position patterns and minimum $\phi$ calculation, (b) Diffraction limit for pattern 1 mirror array position and shifted beam for pattern 3 position with both tilt and vertical levitation motions, (c) Diffraction pattern of tilt only pattern 2 position, which forms a coarse brazed grating. 